\title{
The impact of changing work schedules on American firefighters' sleep patterns and well-being
}

BY LISA M CAPUTO, ALLISON P HAWKES, EMILY E GOSCHE, PETER W VELLMAN, NEALE R LANGE, KRISTIN M SALOTTOLO, RAYMOND CONIGLIO, CHARLES W MAINS

\begin{abstract}
Across the nation, fire departments are adopting the 48/96 work schedule, in which firefighters work 48 consecutive hours with the following 96 hours off. Our study objective was to explain and quantify the impact of switching from the Kelly schedule to the $48 / 96$ schedule by measuring changes in sleep, feelings of daytime function, as well as perceptions of professional and personal well-being for American firefighters. Sleep diaries and self-reported surveys were administered to firefighters at an urban fire department. Sleep diaries measuring the number of hours slept and feelings of refreshment were compared one month before and four months after implementation of the 48/96 schedule. The self-reported surveys measured sleepiness levels via the Epworth Sleepiness Scale. Secondary study objectives included changes in professional and personal well-being time for personal schedules, satisfaction, and health habits before and after the 48/96 schedule was implemented. The 59 firefighters included in the study reported an increase in sleep on-shift after the new schedule implementation (5.8 to 6.6 hours/night, $\mathrm{p}<$ o.oo1). Participants also reported increased feelings of refreshment on days off $(p<0.001)$ and decreased daytime sleepiness $(p<0.001)$. We also found a general trend of improved perceptions of satisfaction, less shift interference with personal schedules and decreased feelings of burnout. American firefighters appeared to benefit from a 48/96 schedule, with short-term
\end{abstract}


improvements in sleep patterns, feelings of burnout, and time for personal schedules.

Key words: firefighters, shift-work sleep disorder, emergency medical technicians, workplace, sleep, fatigue, burnout, professional, organizational culture

\section{Introduction}

The 48/96 schedule, a six-day rotation in which firefighters work 48 consecutive hours followed by 96 hours off, has been widely adopted by American fire departments over the past decade, increasingly supplanting the previously widespread Kelly schedule[1]. The trend toward the 48/96 schedule has resulted in an increased occurrence of four consecutive days off, providing positive externalities such as allowing more full weekends at home, reducing commute days, providing fewer transitions between shifts, and simplifying the scheduling process. On the other hand, the 48 hour on-shift requires firefighters to work a double shift, potentially necessitating 48 consecutive hours of work without sleep, as well as requiring firefighters to be away from families and personal responsibilities for 48 hours.

Firefighters receive an inadequate amount of sleep during 24 hour shifts. (1) This lack of sleep has been found to be an influencing factor in firefighters' psychosocial well-being and fatigue, and has been found to be a major contributor to work stress. (2-4) A study of British firefighters observed a significant decrease in alertness and body temperature and an increase in reaction time over the course of a 24 hour shift. (5)

The impact of work schedules on sleep quality or well-being has rarely been studied in this population. One study reported increases in discipline measures, use of sick leave, traffic and collisions, as well as a slower turn out time, for firefighters on the 48/96 schedule compared with the Kelly schedule. (6) There were more injuries, however, for those working on the Kelly schedule. (6) A review of work schedules noted that of 65 fire departments across the United States that trialed the 48/96 schedule for one year, no departments reverted to previous schedules during or after the year trial period, (7) suggesting that the 48/96 schedule may positively impact firefighters' personal and professional well being. 
Our study aims to explain and quantify the impact of switching from the Kelly schedule to the 48/96 schedule by measuring sleep quality and daytime function, measured by hours of sleep obtained, number of sleep interruptions, time to fall asleep, and feelings of refreshment and daytime sleepiness, reported by firefighters employed by a large, urban fire department. Secondary outcomes of interest included changes in professional and personal well-being, measured as feelings of burnout, time for personal schedules, job satisfaction and health habits. The study was conducted to provide evidence to support a partner fire department's decision to continue the 48/96 schedule, as well as to add to the limited body of knowledge on this topic.

1. The Kelly schedule is a nine-day rotation in which firefighters work three 24 hour shifts with 24 hours off after the first two shifts and 96 hours off after the third shift. 1

\section{Materials and methods}

This pre-post study utilized sleep diaries and self-reported surveys to measure firefighters' sleep quality, daytime function and personal and professional wellbeing before the adoption of the 48/96 schedule, December 2005, and after the schedule change, April 2006. The study was conducted in partnership with an American College of Surgeons verified Level I Trauma Center, and a large urban fire department that also provides Emergency Medical Services. The hospital's Institutional Review Board provided ethical approval of the project. After a brief assembly about the study in December 2005, all personnel (269 members) of the fire department were subsequently sent surveys, sleep diaries, and consent forms in the mail. Responses were submitted on paper and returned to the study coordinator through mail. Consent forms were provided with all surveys, although participants were also invited to complete the form anonymously without signing a consent form. Surveys and consent forms were returned to the Trauma Research Director via prepaid, return envelopes. Data were maintained in a password-protected database.

\section{Survey Instruments}




\section{Sleep diaries}

Sleep diaries were completed after waking each morning for one week before and after implementation of the 48/96 schedule. The printed form contained five questions and was estimated to take less than five minutes to complete per day. In the diaries, participants estimated the amount of time it took to fall asleep, the hours of sleep obtained, the number of sleep interruptions (awakenings) experienced, feelings of refreshment reported on a three-point scale ranging from "fatigued" to "very refreshed," and whether participants were on or off shift the previous night. All sleep diary questions were developed by our research team.

\section{Self-reported survey}

Participants were mailed a separate 37-question self-reported survey before and after implementation of the new work schedule. The eight-question Epworth Sleepiness Scale (ESS) was applied to measure daytime sleepiness levels. Secondary measures of interest included perceived interruptions in personal schedules (five questions); satisfaction (five questions); feelings of burnout (six questions); and health habits (three questions). Age, gender, number of years in current job, whether or not respondent was a paramedic technician, and length of daily commute were also collected. The survey was estimated to take 20 minutes to complete. Non-responders were sent one reminder post card, following the guidance of the Total Design Method. (8)

The ESS is a validated tool that was applied to assess general levels of daytime sleepiness by asking respondents how likely they are to doze off or fall asleep in eight different routine situations during the day. (9) Scores for each question range from zero ("No chance of dozing") to three ("High chance of dozing"), resulting in an overall score of zero to 24 with sleepiness increasing as scores rise. Scores within a range of zero to ten on the ESS are considered normal; scores above ten are considered abnormal and reflect excessive daytime sleepiness. (10) Perceptions of burnout were measured by questions taken from the Copenhagen Burnout Inventory (CBI), a tool developed in 2005 to gauge the general degree of respondents' physical and emotional exhaustion; the tool has been validated among a variety of population. (10-13) Our study applied the six-question client burnout construct, which measured the degree of fatigue and exhaustion that 
participants perceive is due to working with clients. Each question was measured on a five-point scale, in which four indicated the highest degree of burnout. (11) In accordance to the scale, responses were then converted to points on a to 100 scale, in which a response of zero ("To a very low degree") was scored as zero, and four ("To a very high degree") was scored as 100.

Questions gauging available time for personal schedules, satisfaction and health habits were designed by the research team and measured by a five-point likert scale, a seven-point likert scale and open-ended questions, respectively.

\section{Data Analysis}

Our primary outcome of interest was change in sleep quality, measured by hours of sleep obtained, number of sleep interruptions, the time required to fall asleep, and perceptions of daytime function, as measured by a scale of refreshment and scores on the Epworth Sleepiness Scale, pre and post implementation of the 48/96 schedule. Secondary objectives included changes in professional and personal well-being, measured as changes in perceived interruptions in personal schedules due to work schedules, satisfaction, feelings of burnout, and health habits.

Only matched responses to sleep diaries and self-reported surveys were incorporated in the study. Hours of sleep, number of interruptions, and the ESS scores were analyzed using paired sample t-tests to compare change in sleep quality before and after the shift implementation. The results of nominal scales, including the refreshment scale and secondary variables of interest, were described pre and post schedule implementation. There were few missing values; we did not substitute any missing values, thus, the total number of responses varies per question. Analyses were performed using SPSS software (version 21, Chicago, Illinois, USA).

\section{Results}

Of 269 firefighters sent sleep diaries and surveys, a total of 109 and 98 returned sleep diaries (41\% and 36\% response rate) and 160 and 106 returned surveys (60\% and $41 \%$ response rate) in December and April, respectively. Of the sleep diaries, $94 \%$ and $76 \%$ of the total participants signed a consent form in December and 
April, respectively; $76 \%$ and $74 \%$ of total participants chose to sign the consent for the self-reported surveys in December and April, respectively. Fifty-nine participants (22.8\%) completed all surveys and sleep diaries and signed a consent form, and were thereby included in the analysis. There was no significant difference between respondents in the final analysis and all respondents (included those with incomplete datasets); demographic characteristics of participants and the total population are presented in table 1.

\section{Primary Outcomes}

\section{Sleep obtained}

There was a significant increase in mean hours of on-shift nightly sleep, from 5.8 v. 6.6 hours, after the schedule change $(\mathrm{p}<0.001)$. The average number of hours slept per night during off-shift days was similar before and after the new shift was implemented ( 7.2 v. 7.3 hours, $p=0.12$ ). After stratifying the sleep diary results between the first and second 24 hours on-shift, participants slept 26 minutes less during the second 24 hours $(\mathrm{p}=0.06)$ (table 2$)$.

\section{Daytime Sleepiness}

There was a significant decrease in mean scores of daytime sleepiness, as measured by the ESS, from 8.9 to 8.0 (p < 0.001). Ten participants' $(16.9 \%)$ scores reflected a change from abnormal $(\geq 11)$ to normal $(\leq 10)$ levels of sleepiness on the ESS scale; seven participants (11.9\%) switched from normal to abnormal levels of sleepiness during the same period. Over half of participants (33 participants, $55.9 \%$ ) consistently scored within the normal range of sleepiness on the ESS scale, and nine (15.2\%) consistently scored within the abnormal range of sleepiness.

\section{Feelings of refreshment}

For both periods of being on and off shift, there was a considerable improvement in feelings of being refreshed after the 48/96 schedule implementation. During on days, there was a significant improvement in feelings of being refreshed after the 
48/96 schedule implementation ( $\mathrm{p}<0.05)$. Participants felt more fatigued, however, the second half of the 48 -hour shift than the first 24 hours of the shift $(\mathrm{p}<0.05)$. Participants reported a significant improvement in feeling more refreshed on their off days post implementation ( $\mathrm{p}<0.001)$.

\section{Sleep interruptions}

There were no changes in the number of sleep interruptions before and after implementation of the $48 / 96$ schedule (table 2).

\section{Amount of time to fall asleep}

We did not observe any significant changes in the average amount of time to fall asleep pre and post implementation. There was also no appreciable change in time to sleep between the first and second half of the on-shift during the 48/96 shift (table 2).

\section{Secondary outcomes}

\section{Feelings of burnout}

There was a general trend towards reduced burnout after implementation of the new schedule. Specifically, participants were less likely to rate the following perceptions "To a very high degree" or "To a high degree" after implementation: Feeling they gave more than they were getting back when working with patients (19\% v. 9\%, respectively); Feeling tired working with patients (5\% v. 3\%); Feeling frustration when working with patients ( $2 \%$ v. $0 \%)$; Feeling energy drain when working with patients ( $5 \% \mathrm{v}$. 3\%). There was no change in reports of participants wondering how long they could continue working with patients ( $7 \%$ for both).

\section{Personal Schedules}

After implementation, we observed a decrease in the proportion of participants 
that felt the shift interfered with responsibilities. Specifically, there was a decrease in perceptions that the work schedule interfered with performing household activities such as grocery shopping or looking after children (19\% v. 5\%, respectively), participating in leisure activities such as sports or hobbies (16\% v. $3 \%)$, or performing non household activities such as going to doctor or bank $(7 \% \mathrm{v}$. $2 \%)$.

\section{Job Satisfaction}

There was an increase in participants that felt the advantages of the current work schedule outweighed the disadvantages after implementation of the 48/96 schedule (78\% v. $88 \%$, respectively). We did not observe a large shift in questions measuring job satisfaction, including questions regarding satisfaction with the respondents' job, line of work, or perceptions of colleagues' satisfaction with their job or line of work. Additionally, there was not a large shift in responses regarding the frequency of thinking about quitting or the perceived difficulty working with patients. The majority of respondents $(84.7 \%)$ indicated a question gauging spousal support with their work schedule was not applicable.

\section{Health habits}

We found no significant changes in health habits before versus after implementation of the 48/96 schedule. The number of cigarettes smoked per week was unchanged; only one participant reported smoking cigarettes throughout the study. The consumption of alcoholic beverages per week remained unchanged (3.6 both pre and post implementation). The consumption of caffeinated beverages per day also remained constant (2.5 v. 2.2 drinks per day).

\section{Discussion}

American firefighters provide around-the-clock emergency services, necessitating long, uninterrupted work shifts. We found the switch from the Kelly schedule to the $48 / 96$ schedule, led to favorable improvements in sleep, including an increase of hours slept, a small but statistically significant reduction in daytime sleepiness, and an increase in feelings of refreshment. We also observed improved 
scores for secondary objectives of interest, such as feelings of burnout, time for personal schedules and perceptions of satisfaction with the work schedule. The use of alcohol, caffeinated beverages, the number of cigarettes and perceptions of general job satisfaction did not change after the new schedule was implemented.

Cumulative sleep debt has been shown to increase feelings of confusion, mood disturbance, mental exhaustion, stress, deficits in psychomotor vigilance, worsen response inhibition, errors of omission and yield performances comparable to $0.1 \%$ blood alcohol content among other populations. $(14,15)$ One to two nights of recovery sleep have been required to recuperate from these deficits. $(14,16)$ Our finding that there was a small increase in sleep per night on shift may assist in the reduction of fatigue, (17) and may be reflected by increased feelings of refreshment and improvements in feelings of daytime sleepiness. Participatory methods to integrate napping strategies into the work environment may improve with our finding that participants received less sleep the second 24 hours of the 48 hour shift. (18)

Our secondary objective of professional and personal well being perceptions demonstrated an overall trend towards improvement, specifically time for personal schedules and reduced feelings of burnout. These results are in conflict with studies that found long, sporadic work shifts led to difficulty in personal relationships among firefighters, (19) although notably there has not been a similar study examining the effect of the $48 / 96$ schedule, specifically, on personal relationships.

Of note, health habits did not change after implementation of the new schedule. The prevalence of smoking tobacco was lower than other studies of firefighters (only one firefighter in our study reported to smoke cigarettes), although prevalence of smokeless tobacco, which has been found to more common among firefighters than the general population, (20) was not collected. On average, 3.6 alcoholic drinks were consumed per week in our study; this was also lower than other recent epidemiological studies assessing the use of alcohol among American firefighters. $(20,21)$ Lastly, there was a marginal reduction of caffeinated beverage consumption, from 2.5 to 2.2. The numbers remained moderate, demonstrating a continued healthy level of caffeinated beverages through the study.

There are several limitations. First, we had a small sample size, restricting our ability to generalize our findings. As the study allowed participants to either sign a consent form or remain anonymous, we could not match all of the surveys pre 
and post implementation. We had a lower rate of firefighters choosing to sign consent forms in the surveys gauging personal opinions, which may reflect some apprehension about sharing opinions about the shift or their positions. Our results, however, were similar to an unpublished grey paper which included analysis of all respondents in our study population. (22) In our study, we did not include reasons for waking during the night, thus we could not attribute the cause of an awakening as due to a sleep disturbance or due to answering an emergency call. Notably, the fire department received a similar number of calls in December and July (2,997 and 2,898, respectively), and did not expect a large fluctuation in April, but it is not possible to tell the timing of the calls. In any self-reported study, responder bias must be listed as a limitation that may restrict the accuracy of a response. Further, the study period was only one week long, thus we were not able to capture to firefighters' experience throughout the 9 day Kelly shift.

Obesity and heart disease is greatly prevalent within American firefighters, and cardiovascular disease is the leading cause of duty-related death for firefighters. (23) Future studies should examine how shift schedules impact rates of exercise and food choices. Additionally, future studies may wish to utilize a web-based survey application to improve response rates and reduce the time spent sending surveys and diaries in the mail. We would also recommend future studies examine perceptions of safety, including the perception of driving while on shift as well as commuting home after the shift has ended, and include reasons for awakenings. These topics may further contribute to the limited body of knowledge about the impact of work schedules on sleep and satisfaction for firefighters.

\section{Conclusion}

There are few studies that measure the impact of work schedules for firefighters in the United States, and few, if any, publications comparing quality and perceptions of daytime function before and after new shift implementation. Our study provides evidence that participants improved sleep quality and daytime function after switching to the 48/96 schedule from the Kelly schedule. It also improves professional and personal well-being may improve after implementation of the new work schedule. It is our expectation that these findings can be used to guide fire departments across the country in policy debates over work schedules. 
Table 1. Demographic characteristics of participants versus total population.

\begin{tabular}{lll} 
& \multicolumn{2}{c}{ Study Participants $(\mathrm{n}=59)$ Total Participants $(\mathrm{n}=109)$} \\
\hline Male Gender, $\mathrm{n}(\%)$ & $57(96.6)$ & $106(97.2)$ \\
\hline Paramedic, $\mathrm{n}(\%)$ & $28(47.5)$ & $43(39.4)$ \\
\hline Age & $39.5(7.6)$ & $39.8(7.7)$ \\
\hline Years with current job & $13.1(6.3)$ & $13.1(7.1)$ \\
\hline Hours worked per week & $56.1 .(4.9)$ & $56.4(7.3)$ \\
\hline Round trip commute in minutes55.3 $(41.2)$ & $59.8(49.5)$
\end{tabular}

* Denotes findings were statistically significant

Table 2. Sleep measurements for the Kelly Schedule and the 48/69 Schedule.

Work Schedule

Kelly Schedule 48/96 Schedule

Hours of Sleep Obtained per night

\begin{tabular}{lll}
\hline & On Shift 5.8 & $6.6^{*}$ \\
\hline & Off Shift7.2 & 7.3 \\
\hline Number of Interruptions & & \\
\hline & On Shift 2.0 & 1.8 \\
\hline Amount of Time to Fall Asleep & Off Shift1.2 & 1.1 \\
\hline & On Shift 17.8 & 16.9 \\
\hline & Off Shift17.9 & 19.9
\end{tabular}

* Denotes findings were statistically significant

\section{References}

1. Harvey B. Firefighter Sleep Deprivation in the Raleigh (NC) Fire Department: An Objective Test. Fire Eng 2013 Feb 1.

2. Kim KH, Kim JW, Kim SH. Influences of Job Stressors on Psychosocial Well-being, Fatigue and Sleep Sufficiency among Firefighters. Korean J Occup Environ Med 2006 Sep 1;18(3):232-45.

3. Domrachev AA, Savchenkov YI, Mikhailova LA. Some Personality Characteristics of Fire Service Specialists under Conditions of Prolonged Intense Workloads. Hum Physiol 2004 Sep 1;30(5):581-4. 
4. Murphy SA, Beaton RD, Cain K, Pike K. Gender Differences in Fire Fighter Job Stressors and Symptoms of Stress. Women Health 1995;22(2):55-69.

5. Knauth P. Changing schedules: Shiftwork. Chronobiology International [serial online] 1997 [cited 2013 Oct 1];:Vol. 14 No. 2:159-71. Available from:URL:http://informahealthcare.com/doi/abs/10.3109/07420529709001153

6. James Clack. The management effects of firefighters working a consecutive 48hour shift. An applied research project submitted to the National Fire Academy as part of the Executive Fire Office Program. 2003 Feb.

7. Downey Firemen's Association. Proposal for the 48/96 work schedule [serial online]. 2008. Available from: URL:http://www.dfalocal3473.org/4896.doc

8. Dillman DA. Mail and Telephone Surveys: The Total Design Method. New York: Wiley; 1978.

9. Johns M. 1997 Version of ESS [serial online]. The Epworth Sleepiness Scale. [cited 2013 Oct 20]. Available from: URL:http://epworthsleepinessscale.com/1997version-ess/

10. Johns M, Hocking B. Excessive daytime sleepiness: daytime sleepiness and sleep habits of Australian workers. Sleep 1997;20(10):844-9.

11. Kristensen TS, Borritz M, Villadsen E, Christensen KB. The Copenhagen Burnout Inventory: A new tool for the assessment of burnout. Work Stress 2005;19(3):192207.

12. Yeh WY, Cheng Y, Chen CJ, Hu PY, Kristensen TS. Psychometric properties of the chinese version of copenhagen burnout inventory among employees in two companies in Taiwan. IntJ Behav Med 2007 Sep 1;14(3):126-33.

13. Milfont TL, Denny S, Ameratunga S, Robinson E, Merry S. Burnout and Wellbeing: Testing the Copenhagen Burnout Inventory in New Zealand Teachers. Soc Indic Res 2008 Oct 1;89(1):169-77.

14. Dinges DF, Pack F, Williams K, Gillen KA, Powell JW, Ott GE, et al. Cumulative sleepiness, mood disturbance, and psychomotor vigilance performance decrements during a week of sleep restricted to 4-5 hours per night. Sleep 1997 Apr;20(4):267-77.

15. Williamson AM, Feyer AM. Moderate sleep deprivation produces impairments in cognitive and motor performance equivalent to legally prescribed levels of alcohol intoxication. Occup Environ Med 2000 Oct;57(10):649-55.

16. Drummond SPA, Paulus MP, Tapert SF. Effects of two nights sleep deprivation and two nights recovery sleep on response inhibition. J Sleep Res 2006 Sep;15(3):261-5.

17. Takeyama H, Itani T, Tachi N, Sakamura O, Murata K, Inoue T, et al. Effects of shift schedules on fatigue and physiological functions among firefighters during night 
duty. Ergonomics 2005;48(1):1-11.

18. Takeyama H, Kubo T, Itani T. The Nighttime Nap Strategies for Improving Night Shift Work in Workplace. Ind Health 2005;43(1):24-9.

19. Shakespeare-Finch J, Smith S, Obst P. Trauma, coping resources, and family functioning in emergency services personnel: A comparative study. Work Stress 2002;16(3):275-82.

20. Poston WS, Haddock CK, Jahnke SA, Jitnarin N, Day RS. An examination of the benefits of health promotion programs for the national fire service. BMC Public Health 2013 Sep 5;13(1):805.

21. Haddock CK, Jahnke SA, Poston WSC, Jitnarin N, Kaipust CM, Tuley B, et al. Alcohol use among firefighters in the Central United States. Occup Med Oxf Engl 2012 Dec;62(8):661-4.

22. Hawkes A. Evaluation of the 48-96 Schedule for West Metro Fire Rescue. 2006.

23. Fahy RF. US firefighter fatalities due to sudden cardiac death, 1995-2004. National Fire Protection Association; 2005.

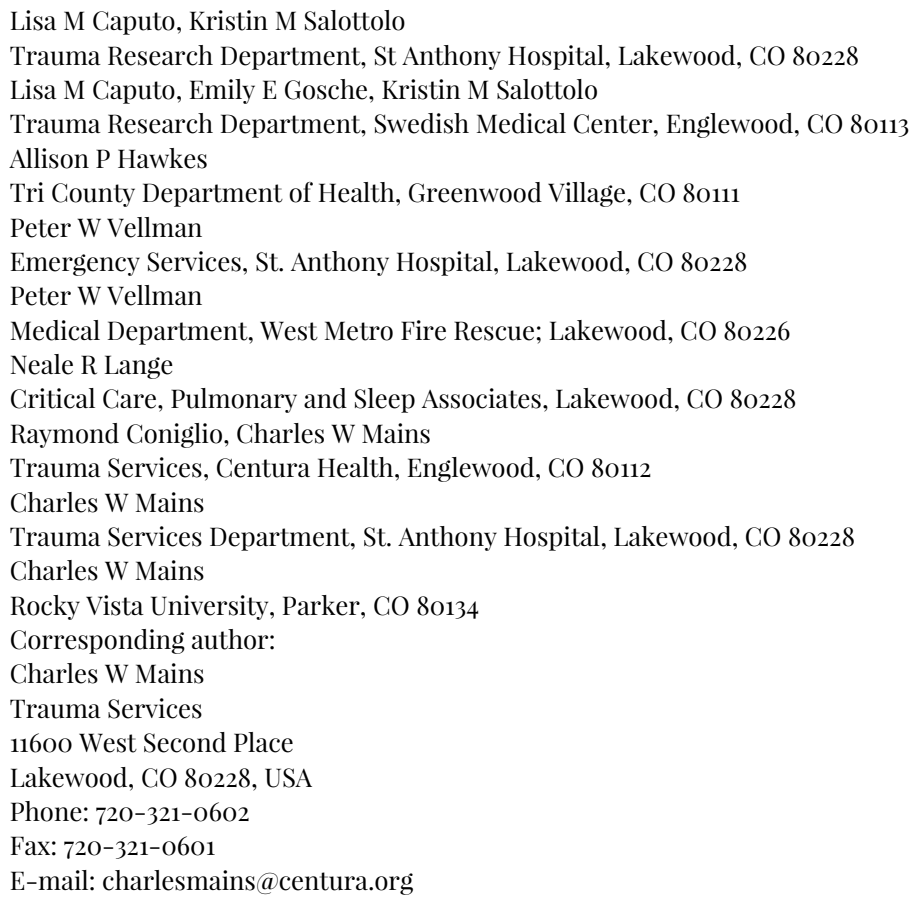

